\title{
High-precise transient response model of semiconductor gas sensor considering temperature dependency of carrier mobility
}

\author{
A. Fujimoto ${ }^{1}$, M. Kita ${ }^{1}$, Y. Katoh ${ }^{1}$ \\ ${ }^{1}$ Wakayama National College of Technology, 77 Noshima, Nadacho, Gobo-shi 644-0023 Japan, \\ fujimoto@wakayama-nct.ac.jp
}

\begin{abstract}
The model of transient response of $\mathrm{SnO}_{2}$ semiconductor gas sensor under modulation heating has been constructed and improved successfully to reveal the forming process of the transient response. The transient responses were estimated by carrier concentrations determined from oxygen concentrations calculated by the model. The model made clear that activation energy of the chemical reaction affected strongly to the transient response of the sensor. Calculated transient responses using activation energies determined by molecular orbital calculations had deviated from experimental results. Temperature dependency of electron mobility in $\mathrm{SnO}_{2}$ was introduced newly to the model to decrease the deviation. The deviation could be decreased 1/10 at the sum of square. New precise model of transient response have been constructed by considering the temperature dependency.
\end{abstract}

Key words: Gas sensor, $\mathrm{SnO}_{2}$, Transient response, Temperature dependency, Carrier mobility

\section{Purpose and Background}

A realization of smell selective gas sensor is desired for variety application field such as gas leakage detection alarms, food production controls and so on. $\mathrm{A} \mathrm{SnO}_{2}$ gas sensor is used widely for gas leakage alarms in home due to high sensitivity, stability and substantiality of the sensor. On the other hand, low selectivity of the sensor for kind of gases cause to malfunction of the alarm. Early improvements of the selectivity are desired.

It was known that the transient responses of the sensor differed for different gases ${ }^{1)}$. We have tried to realize the sensor by using the differences of the transient response shown in figure 1. Calculation model of transient response of the sensor was developed to make

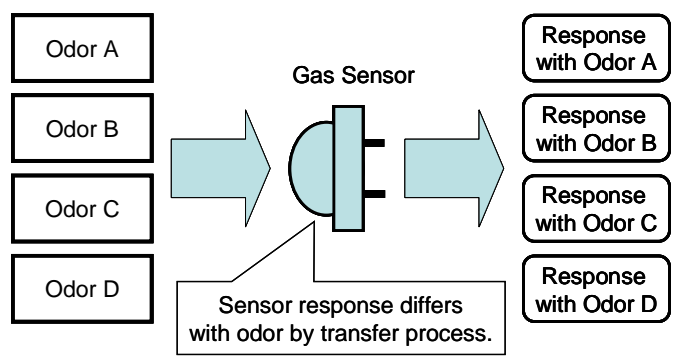

Fig.1 Sensor transient response with different odors. clear the forming process of the transient responses and to predict the responses from characteristics of the gases without any experiments.

The calculated transient responses of the sensor by using the model were agreed well with experimental results ${ }^{2}$ and strongly depended on activation energy of chemical reaction on the sensor surface between inflammable gases and adsorbed oxygen on the sensor surface ${ }^{3)}$. We have tried to estimate the activation energies by molecular orbital calculation because of the accurate values of the activation energies at chemical reaction of oxygen and almost all inflammable gases were

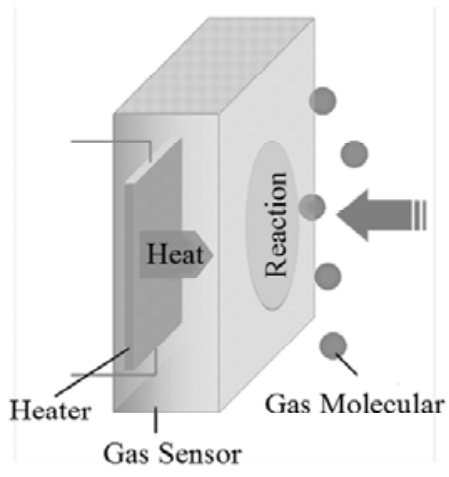

Fig. 2: Schematic of gas sensor for modeling. 
not reported till now.

Calculated transient responses had deviated from experimental results by using activation energies estimated from the molecular orbital calculations. The model was improved by introducing the temperature dependency of electron mobility in $\mathrm{SnO}_{2}$.

\section{Outline of the Transient Response Model ${ }^{2)}$}

Thick film type gas sensor shown in figure 2 was assumed for the modeling. Electric heater and sensor materials are placed on opposite side of the surface on the ceramic substrate. When applying a current to the heater, the temperature of the heater goes up and the heat transfers to the sensor through the substrate. The sensor temperature was determined by the heat conduction, heat dispersion by electromagnetic radiation and air cooling.

Chemical reaction between oxygen adsorbed on the sensor surface and inflammable gas included in the air will progresses with the temperature rise of the sensor. The concentration of the inflammable gas decreases with progresses the chemical reaction and the gas diffuses from the ambient to the sensor surface by concentration gradient of the gas. These processes are shown schematically in figure 3. Carrier concentration and sensor response are calculated from the oxygen concentration on the sensor surface.

\section{Estimation of the Activation Energy by Molecular Orbital Calculation ${ }^{3)}$}

The tin oxide cluster consisted of 54 tin atoms and adsorbed oxygen atom on (110) surface was made by WinMOPAC package. The inflammable gas molecular are located above the (110) surface of the cluster shown in figure 4. The total heat of formation of the whole system was calculated with different distance between the cluster surface and inflammable gas molecular by PM5 Hamiltonian.

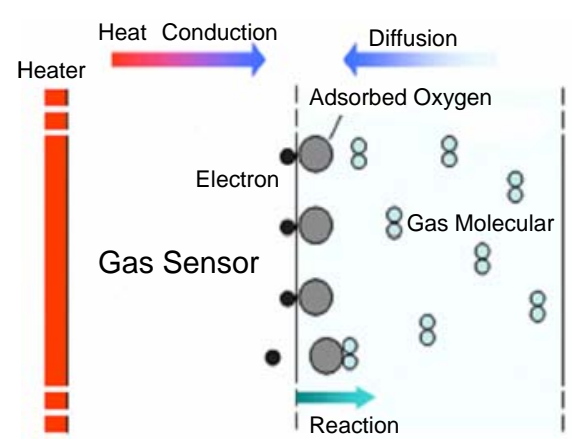

Fig.3 Calculation model for transient response of semiconductor gas sensor.

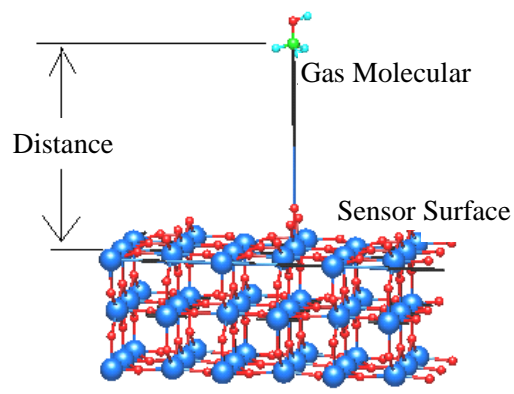

Fig. 4 Configuration of the calculation with tin dioxide cluster and gas molecular.

Figure 5 shows the total heat of formation change with changing the distance from the cluster surface to methanol molecular as an inflammable gas. The total heat of formation increase gradually with closing the methanol molecular to the surface and reaches maximum value at the distance of around 4 angstroms. The value decreases steeply with decreasing the distance and reaches bottom at around 3.8 angstroms. Chemical reactions of the inflammable gas and the oxygen atom will occur around the distances. The activation energy of the reaction were defined to energy difference between the peak value of heat of formation with the distance of 4 angstroms and the value with the distance of enough large shown in figure 5.

\section{Temperature Dependency of Carrier Mobility}

Carrier mobility in semiconductor decreases with increasing temperature by lattice vibration. The electron mobility of $\mathrm{SnO}_{2}$ has been treated as a constant value for simplify the model even the mobility changes by temperature. We tried to make a new version of the model which took into account the temperature dependency of the mobility to improve the accuracy. Figure 6 shows the typical temperature dependency of the electron mobility in $\mathrm{SnO}_{2}$. The dependency was approximated as $\mu=70734 \times T^{2.535}$ for calculation. This relation was incorporated into

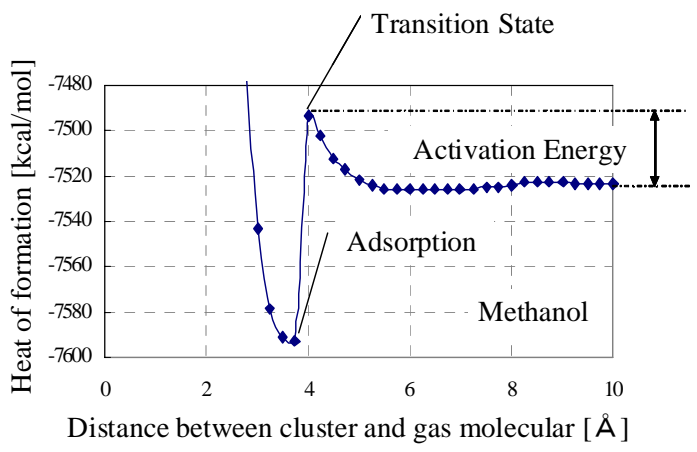

Fig. 5 Calculated total heat of formation for various distance from cluster to methanol molecular. 


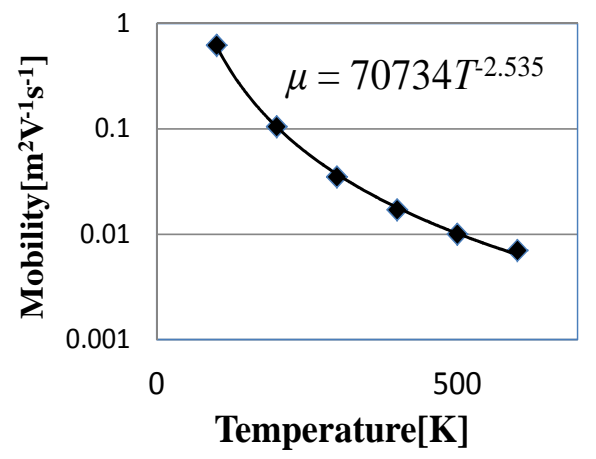

Fig. 6 Temperature dependency

the calculation process of the model of transient response. Transient responses with thermal modulation were calculated and compared to experimental output.

Calculated transient responses with and without temperature dependency of the electron in $\mathrm{SnO}_{2}$ are shown in figure 7 together with experimental result. The calculated response with temperature dependency of electron is closer to experimental response than the response without the dependency. The deviation from experimental response could be decreased $1 / 10$ at the sum of square. New precise model of transient response have been constructed successfully by considering the temperature dependency of electron mobility in $\mathrm{SnO}_{2}$.

\section{Conclusions}

The model of transient response of semiconductor gas sensor under modulation heating has been constructed and improved successfully. Temperature dependency of electron mobility in $\mathrm{SnO}_{2}$ was introduced to the model to decrease the deviation from experimental response. The deviation could be

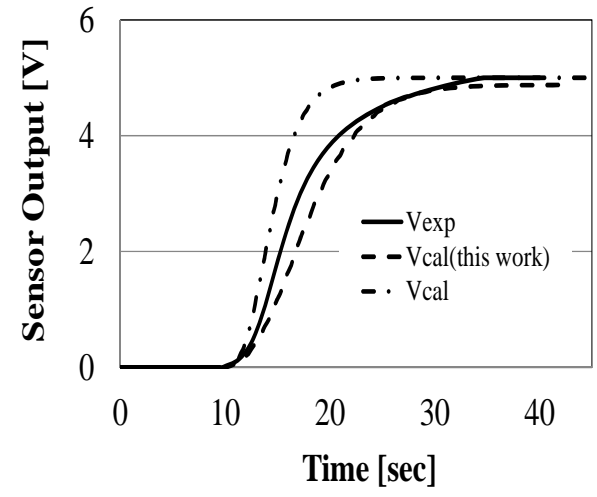

Fig. 7 Calculated transient response with (broken) and without (dashed) temperature dependency of carrier mobility together with experimental response (solid).

decreased $1 / 10$ at the sum of square by taking into account the temperature dependency of electron mobility.

The new precise model of transient response will become a powerful tool to predict the transient responses of $\mathrm{SnO}_{2}$ gas sensor under modulation heating for any inflammable gases without experiments. On the contrary, it will become possible to identify the kind of gas from transient response using this model.

\section{References}

[1] A.Fujimoto: "Response of smell sensor for alcohol gases", 8th International Meeting on Chemical Sensors, Basel Switzerland, July 2000 , Abstract Book p.494.

[2] A. Fujimoto, M. Tomatani and T. Kuwahara; "Model of Transient Response of Semiconductor Gas Sensor", Sensor Letters, Vol. 6, No. 6, pp. 883-886 (2008).

[3] A. Fujimoto, J. Ohsumi, and T. Ohtani:" Activation Energy Dependence on Transient Response of Semiconductor Gas Sensor", Sensor Letters, Vol.9, No.2, pp.732-735 (2011). 